\title{
Effectiveness of reverse total shoulder arthroplasty for primary and secondary fracture care: mid-term outcomes in a single-centre experience
}

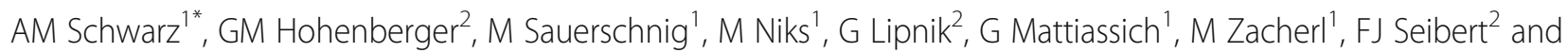
$\mathrm{M} \mathrm{Plecko}^{1}$

\begin{abstract}
Background: The introduction of reverse total shoulder arthroplasty (RSA) as a treatment option in complex proximal humeral fractures, has significantly extended the surgical armamentarium.

The aim of this study was to investigate the mid-term outcome following fracture RSA in acute or sequelae, as well as salvage procedures. It was hypothesized that revision RSA (SRSA) leads to similar mid-term results as primary fracture treatment by RSA (PRSA).

Methods: This retrospective study describes the radiological and clinical mid-term outcomes in a standardized single-centre and Inlay design. Patients who underwent RSA in fracture care between 2008 and 2017 were included (minimum follow-up: 2 years, minimum age: 60 years).

The assessment tools used for functional findings were range of motion (ROM), Visual Analogue Scale, absolute (CS) plus normative Constant Score, QuickDASH, and Subjective Shoulder Value. All adverse events as well as the radiological results and their clinical correlations were statistically analysed (using $p<.05$ and $95 \%$ confidence intervals).

Results: Following fracture RSA, 68 patients were included (mean age: 72.5 years, mean follow-up: 46 months). Forty-two underwent primary RSA (PRSA), and 26 underwent revision RSA (SRSA). Adverse advents were observed in $13 \%(n=9 / 68)$.

No statistically significant results were found for the scores of the PRSA and SRSA groups, while the failed osteosynthesis SRSA subgroup obtained statistically significantly negative values for ROM subzones (flexion: $p=.020$, abduction: $p=.020$ ). Decreased instances of tubercle healing were observed for the in PRSA group relative to the SRSA group $(p=.006)$. The absence of bony healing of the tubercles was related to significant negative clinical and subjective outcomes (all scores: $p<.05$, external rotation: $p=.019$ ). Significant postoperative improvements were evaluated in the SRSA group (CS: 23 to 56 at mean, $p=.001$ ), the time from index surgery to operative revision revealed no associations in functional findings.

(Continued on next page)
\end{abstract}

\footnotetext{
* Correspondence: angelika.schwarz@auva.at

${ }^{1}$ AUVA - Trauma Hospital (UKH) Styria | Graz, Teaching Hospital of the

Medical University of Graz, Göstinger Straße 24, 8020 Graz, Austria

Full list of author information is available at the end of the article
}

(c) The Author(s). 2021 Open Access This article is licensed under a Creative Commons Attribution 4.0 International License, which permits use, sharing, adaptation, distribution and reproduction in any medium or format, as long as you give appropriate credit to the original author(s) and the source, provide a link to the Creative Commons licence, and indicate if changes were made. The images or other third party material in this article are included in the article's Creative Commons licence, unless indicated otherwise in a credit line to the material. If material is not included in the article's Creative Commons licence and your intended use is not permitted by statutory regulation or exceeds the permitted use, you will need to obtain permission directly from the copyright holder. To view a copy of this licence, visit http://creativecommons.org/licenses/by/4.0/ The Creative Commons Public Domain Dedication waiver (http://creativecommons.org/publicdomain/zero/1.0/) applies to the data made available in this article, unless otherwise stated in a credit line to the data. 
(Continued from previous page)

Conclusions: RSA is an effective option in severe shoulder fracture management with predictable results for salvage as well as first-line treatment. Promising mid-term functional results, reasonable implant survival rates, and high patient satisfaction can be achieved.

Level of evidence: Level III.

Keywords: Reverse total shoulder arthroplasty, Complex shoulder fracture care, Primary fracture treatment, Primary fracture reverse total shoulder arthroplasty, Secondary fracture treatment, Secondary fracture reverse total shoulder arthroplasty, Salvage procedure, Tubercle bone stock healing

\section{Background}

Proximal humeral fractures represent the third most common fracture type in the elderly population $[1,2]$. The management of these injuries remains challenging due to demographic changes, as well as the simultaneous incidence of osteoporosis, and various other comorbidities [1]. The spectrum of surgical treatment modalities include closed reduction and percutaneous pin fixation [1], open reduction and internal fixation (ORIF) by locking or non-locking plates [3-7] and intramedullary nails $[8,9]$. Further primary non-joint-preserving treatment options for proximal humeral fractures include anatomical total shoulder arthroplasty [10-13], shoulder hemiarthroplasty [14], and reverse shoulder arthroplasty (RSA) [13, 15-18]. However, the functional results tend to be inferior in anatomical total shoulder arthroplasty and shoulder hemiarthroplasty due to rotator cuff deficits or unsuccessful tubercle refixation in cases of fracture $[10,19,20]$.

Meanwhile, recent trends show that RSA has become the treatment of choice for complex proximal humeral fractures, especially in patients with poor bone quality [21]. The RSA was primarily designed to treat patients with massive rotator cuff defects [22-25]. However, indications have been extended to further pathologies, such as cuff tear arthropathy $[26,27]$, and proximal humeral fractures [17, 28] or revision arthroplasty [29]. Moreover, RSA is used as a salvage procedure in cases of symptomatic mal- or nonunions following (failed) primary osteosynthesis or shoulder hemiarthroplasty of proximal humeral fractures $[1,13,29]$.

The goal in clinical practice is to perform a stable osteosynthesis after meticulous, gentle reduction without denudation of fracture fragments in complex fracture situations. Various treatment options and expectations for acute proximal humeral fractures have led to vivid discussions between surgeons. To address this focus, we hypothesized that a failed joint preserving strategy treated by revision RSA might achieve similar clinical results as an approach utilizing primary joint replacement. Therefore, the aim of this study was to evaluate the functional outcome following primary RSA (PRSA) for proximal humeral fractures compared to secondary RSA (SRSA) performed as a salvage procedure.

\section{Methods}

\section{Study design and patient recruitment}

This retrospective case-series study characterized a single-centre evaluation in a standardized setting following reverse total shoulder arthroplasty in primary (PRSA) or secondary / sequelae (SRSA) fracture care.

All consecutive patients older than 60 years at the time of surgery were included after the treatment by either PRSA or SRSA between January 2008 and December 2017 at a level-III trauma centre (AUVA - Trauma Hospital (UKH) Styria | Graz). Figure 1 shows the years of RSA implantation (in three periods) of all included patients. The timeline was chosen based on data accessibility. Only patients with the Inlay (Grammont) design $\left(155^{\circ}\right.$ humeral neckshaft angle) were included; two different implants were involved (Delta Xtend, DePuy Synthes; Warsaw, USA and Anatomical Reverse, Zimmer; Warsaw, USA\}.

All included patients had received RSA implantation as a primary or secondary treatment following three- or four-part fractures (types 4 and 5) and fracturedislocations (type 6) according to the classification by Neer [30, 31]; see Fig. 2 for detailed distributions. In the PRSA group, all cases were treated with a reverse shoulder arthroplasty within ten days of trauma. For the SRSA group, a minimum time interval of three months from index to revision surgery was defined. The SRSA group was comprised of two subgroups of patients that had undergone RSA implantation in cases of failed ORIF or following all otherwise non-implant related fracture treatment adverse events. See Fig. 3 for the detailed study design.

\section{Patient characteristics}

Data were collected prospectively in the respective hospital's database. Patient characteristics and pathological as well as course of treatment data were collected and analysed retrospectively. All postoperative adverse events were evaluated via the hospital's database and the patients' medical histories were evaluated in the final patient examination. Major complications were specified as events requiring an unplanned revision, all others were 


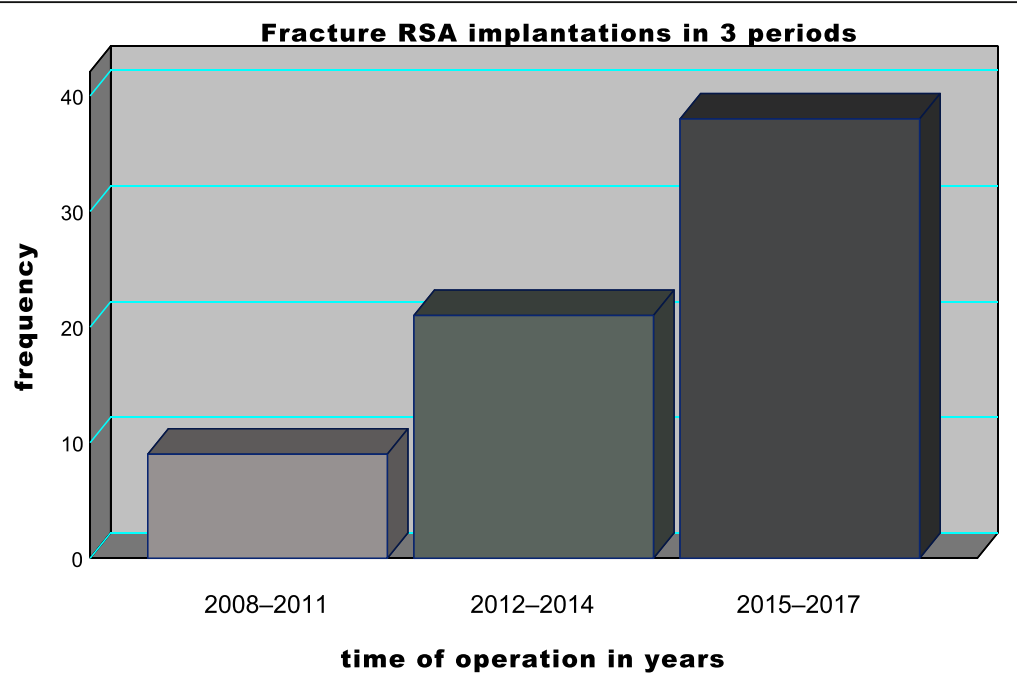

Fig. 1 Fracture RSA implantations by years: The bar chart displays the operation time of each year in 3 time intervals. A constant increase in fracture RSA rates of implantation is shown. (RSA - reverse total shoulder arthroplasty)

classified as minor complications. The follow-up time was defined as the interval between surgery and the last assessment. The minimum follow-up was fixed at two years.

\section{Specific details of the operative and postoperative procedures}

The operative procedure and rehab protocol were the same for the entire sample population and were in accordance with the standardized work-up at our centre. A deltopectoral approach was performed in the beachchair position in every case. Further, all humeral monobloc components were cemented. A full $360^{\circ}$ release was accomplished under axillary nerve visualization for glenoid preparation If necessary, an additive arthrolysis and scar release were performed.

All tubercles were fixed via non-resorbable transosseous and cerclage sutures and circular sutures around the prosthesis neck. If a detachment of the subscapularis tendon was necessary, a double-row transosseous refixation was implemented. Passive physiotherapy with a free range of motion $(\mathrm{ROM})$ in a pain-free interval was started 2 days postoperation. The active-assistive motion was initiated five weeks post-operation, and deltoid muscle mass improvement was fostered at the beginning of the seventh week.

\section{Proximal humeral fractures according to the classification of Neer}

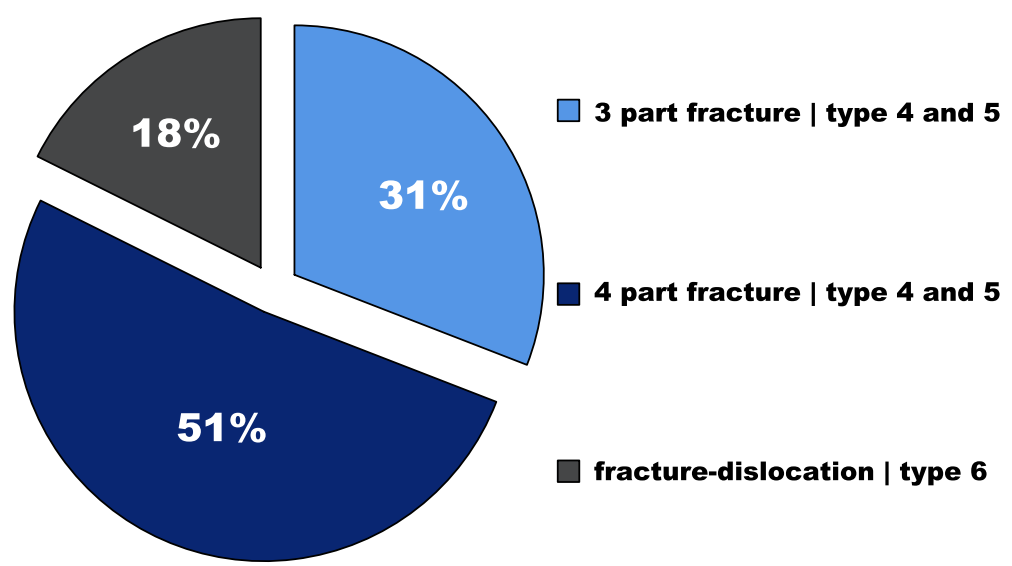

Fig. 2 Fracture classification: The circle chart reports the distribution in percentages of all included patients based on the classification proposed by Neer. $[30,31]$ 


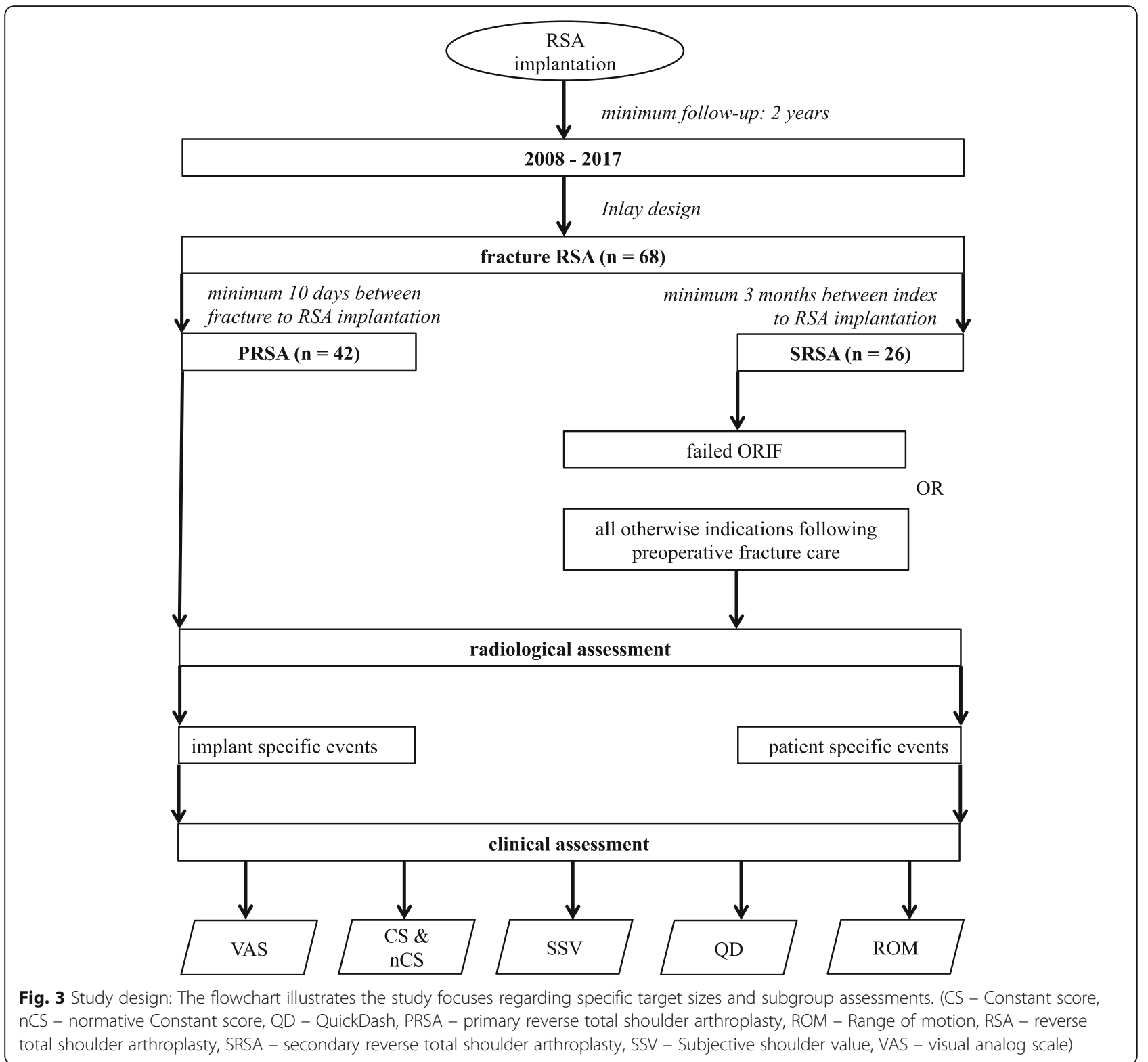

\section{Clinical outcome assessment}

Patients were assessed to determine their current clinical level via the following scores: Visual Analog Scale (VAS), absolute Constant Score (CS) [32], normative Constant Score (nCS) [33], Subjective Shoulder Value (SSV) [34], and QuickDASH (QD) [35]. ROM was evaluated in degrees of flexion, abduction, and external rotation (ER). The internal rotation (IR) was characterized in points based on the functional shoulder-specific CS [32]. Patient-specific assessment was carried out via the modified valuation of the CS, which is based on age- and gender-related characteristics (nCS) [33].

The SSV is a Single Assessment Numeric Evaluation (SANE) of the shoulder and represents a shoulder selfassessment by the patient. The score is expressed as a percentage of an entirely healthy shoulder, which would score $100 \%$ [34]. The QD is a self-assessment instrument and includes eleven questions concerning complaints regarding the upper extremity and activities of daily living [35]. The preoperative CS, which was available in their prospective documentation in the hospital's database, was compared to their respective postoperative values.

\section{Specific targets of clinical outcomes}

Further analysis of the data from the SRSA group involved considering the mid-term CS outcomes for early ( $<12$ months) and late (>12 months) time intervals from index surgery to revision RSA implantation.

Additionial analysis of the entire collective regarding their CS outcomes was carried out by consideration of 
mid-term periods (two - five years) and longer-term periods (more than five years). See Fig. 1 for detailed RSA implantation data (displayed in years).

\section{Radiological outcome assessment}

During follow-up, X-rays were performed in three planes (anterior-posterior, axial, and supraspinatus outlet view). The final X-rays were analysed and compared to the index and interim radiological data. These data were evaluated by three trauma and orthopaedic surgeons (AS, GH, and $\mathrm{MN}$ ) for implant dislocation, grade of notching according to Sirveaux [36], healing of the major and/or minor tubercles, and radiological signs of loosening of the prosthesis.

Anatomic tubercle healing was defined by visible tubercles on the lateral and anterior part of the stem, in continuity with the diaphysis and at the level below or the same level as the top of the humeral implant (see Fig. 4). Non-anatomic healing was specified as malunion, nonunion, or resorption (see Fig. 5).

All radiological analyses were defined via raters' consensus, which means a full agreement of all three observers in all target sizes. To assess the clinical correlations with the radiological outcomes, the CS and
nCS were chosen for evaluation in the following focuses: tubercle bone stock healing and scapular notching.

\section{Statistical analysis}

Statistical analysis was performed using the software SPSS \{IBM SPSS Statistics version 26, Armonk, USA\}. Continuous parameters are presented as means, standard deviations (SD), and categorical or quantitative data.

In order to compare findings between the PRSA and SRSA groups, a t-test was utilized. The Kruskal-Wallis and post-hoc Dunn-Bonferroni methods were used to interpret the data between the PRSA and both SRSA subgroups - see Fig. 3 for an illustration of the study design). The Spearman's Rho correlation coefficient $(\rho)$ was additionally used for the final relationship analyses. A Fisher's exact test was utilized to compare the rates of adverse events. P-values $(p)$ below 0.05 were considered as statistically significant, and confidence intervals of 95\% were computed.

\section{Results}

Demographics

In total, 68 patients met the inclusion criteria. In 58 cases a Delta Xtend \{DePuy Synthes; Warsaw, USA\}
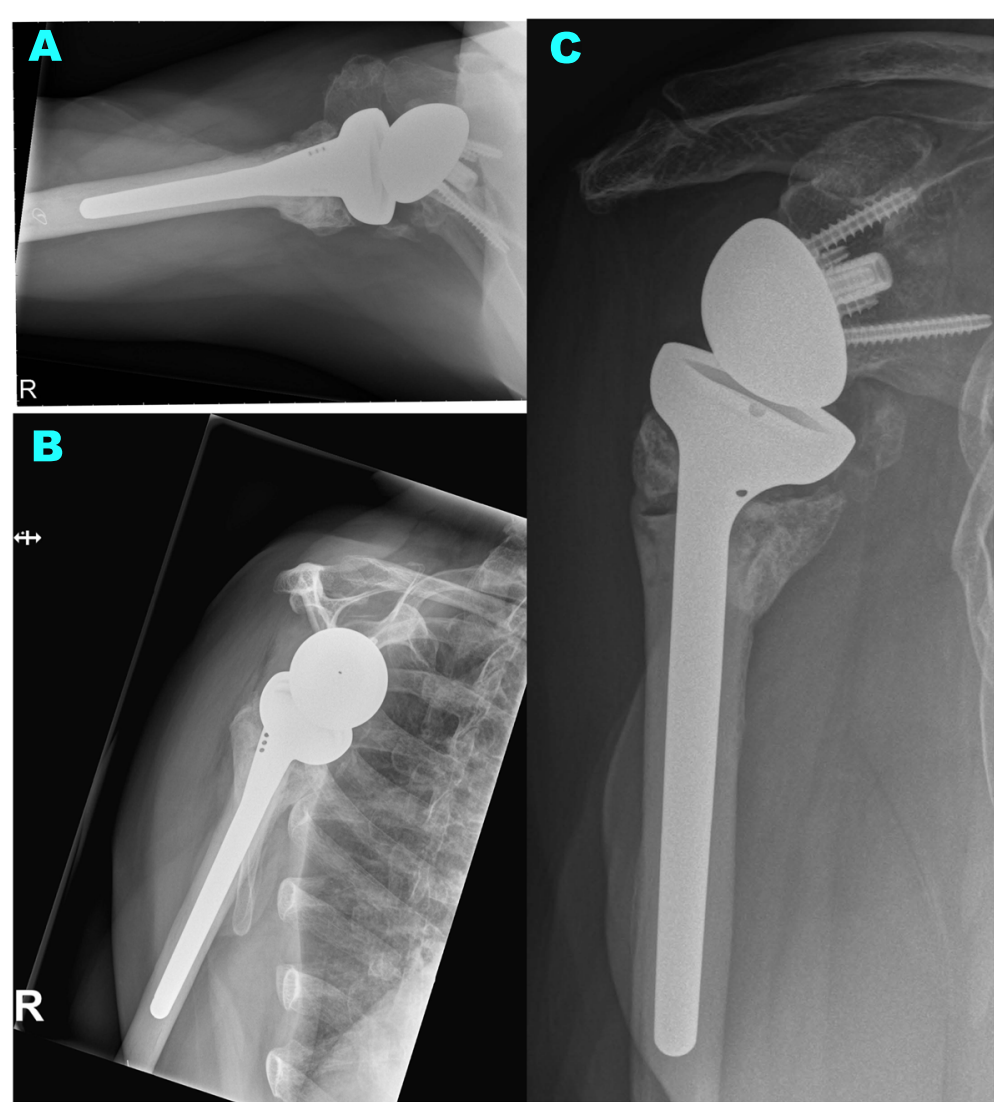

Fig. 4 Anatomic healing of the tuberosities: A case of a 73 years old woman, an X-ray is pictured in three planes 60 months post operation. (a: axial view, b: supraspinatus outlet view, c: anterior-posterior view) 


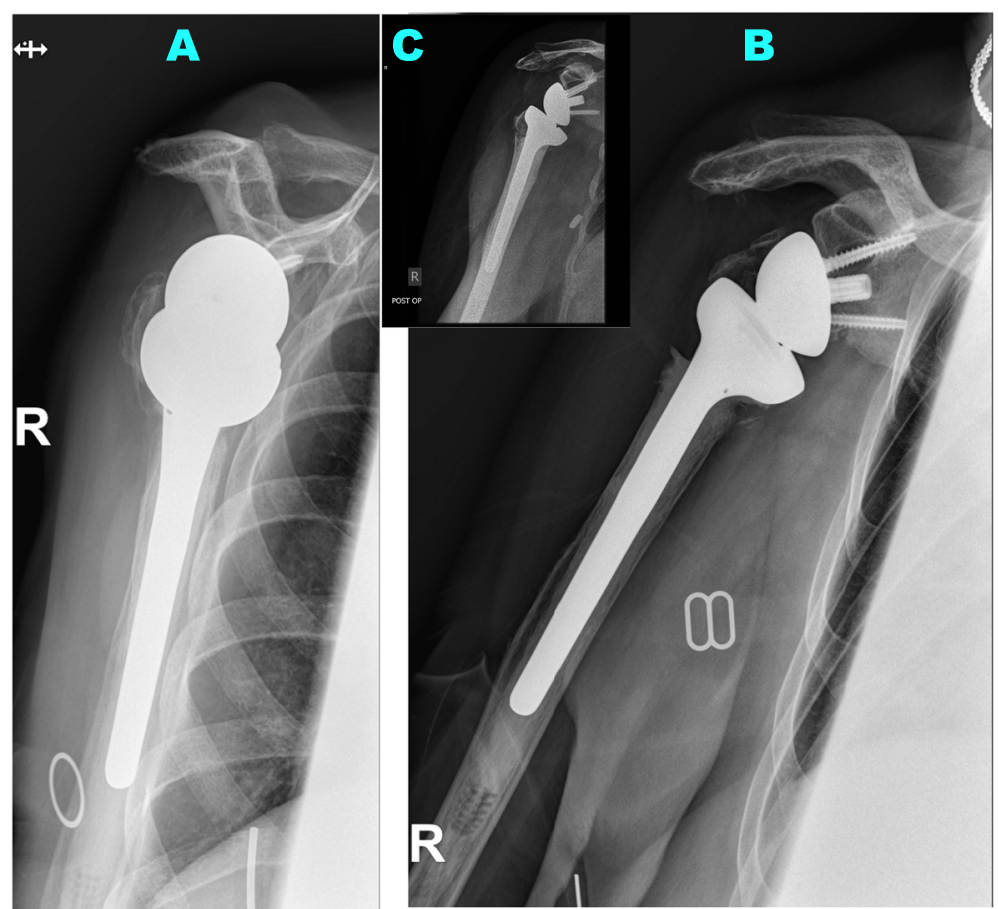

Fig. 5 Malunion of the greater tuberosity: A case of a 78 years old woman, a X-ray is pictured in three planes 35 months post operation. A post operation index $\mathrm{X}$-ray (c) is added for comparision of the greater tubercle to final radiological outcome. (a: supraspinatus outlet view, $\mathbf{b}$ : anteriorposterior view, c: anterior-posterior view in $\mathrm{X}$-ray two days post operation)

system was utilized, and in ten cases an Anatomical Reverse $\{$ Zimmer; Warsaw, USA system was utilized. The PRSA group consisted of 42 patients, and the SRSA group consisted of 26 patients. The SRSA collective was comprised of ten patients $(39 \%, n=10 / 26)$ who were treated following failed ORIF in the first subgroup. The second subgroup $(61 \%, n=16 / 26)$ consisted of twelve cases $(46 \%, n=12 / 26)$ who were treated after humeral head necrosis and four patients $(15.5 \%, n=4 / 26)$ who were undergoing primary trauma hemiarthroplasty.

The mean age of the entire collective was 72.5 years (SD: 6.8, range: 60-89) at the time of surgery; 73.6 years (SD: 6.8) in the PRSA group and 70.5 years (SD: 6.7) in the SRSA group. The average follow-up time for all patients was 46 months (SD: 25.1, range: 24-134); 41 months (SD: 21.3) in the PRSA group and 54 months (SD: 26.5) in SRSA group. The mean surgery time duration was 160 minutes (SD: 44.1) for the PRSA group, 207 minutes (SD: 38.2) in for the SRSA group, and 178 minutes (SD: 47.6, range 86-352) for all patients. Surgical revision was performed at an average of 45.5 months (SD: 54.1, range: 3.4-180) following index surgery in the SRSA group.

\section{ROM}

All average ROM values were comparable in each of the groups. No statistically significant difference was found between the PRSA group versus the SRSA group (all P- values $\geq 0.05$ ). When we compared the SRSA subgroups and the PRSA group, statistically significant negative average flexion $(p=.020)$ and abduction $(p=.020)$ values were found for patients who were previously treated following failed ORIF. The adjusted post-hoc test validated these significant results. All specific ROM values are discussed in Table 1; Fig. 6a-d. In analysing the ROM results, the strongest relationship was found for flexion and abduction with a correlation coefficient of $\rho=0.901$ $(p<.001)$.

\section{Scores}

The outcome values of the PRSA and SRSA groups did not reveal any statistically significant differences, as displayed in Table 2. Thus, no significant differences were observed in CS $(p=.204)$ and nCS $(p=.211)$. Similarly, no significant associations were found for both scores when comparing the values of the PRSA group with the values of the individual SRSA subgroups (see Fig. $7 \mathrm{~b}$ for CS).

When comparing the VAS and QD scores for primary and secondary care, homogeneous results were observed for the PRSA and SRSA groups $(p=.573$ and 0.291), as shown in Table 2. No statistically significant results were found regarding the VAS and QD scores for the SRSA subgroups ( $p \geq .05$, see Fig. 7a and c). Equivalent SSV results were found for the PRSA and SRSA groups $(p=$ 
Table 1 ROM of PRSA versus SRSA

\begin{tabular}{llll}
\hline ROM & PRSA & SRSA & $P$-value \\
\hline Flexion & $125^{\circ}($ SD: 39.6) & $122^{\circ}$ (SD: 35.3) & 0.598 \\
Abduction & $118^{\circ}$ (SD: 38.2) & $107^{\circ}$ (SD: 32.3) & 0.184 \\
ER & $17^{\circ}($ SD: 18.7) & $14^{\circ}$ (SD: 17.8) & 0.552 \\
IR & 4 (SD: 2.6) & 3 (SD: 1.9) & 0.169 \\
\hline
\end{tabular}

No statistically relevant difference was observed between the PRSA and SRSA groups; good results were obtained for both groups. Flexion, abduction, and ER are reported in degrees, and IR in points according to a sub-item of the Constant Score [32]. The average values are presented

(ER external rotation, $I R$ internal rotation, $R O M$ range of motion, PRSA primary reverse total shoulder arthroplasty, SRSA secondary reverse total shoulder arthroplasty, SD standard deviation)

$.558)$, see Fig. $7 \mathrm{~d}$. Of the score data analysed, the most substantial relationship was found for the CS and QD with a correlation coefficient of $\rho=-0.785(p<.001)$.

A significant improvement in CS was observed in the SRSA group; the preoperative mean CS was 23 (SD: 9.7), and the postoperative average CS was 56 (SD: 13.9) in the final follow-up $(p=.001)$, see Fig. 8. Additionally, SRSA differentiation in the early ( $<12$ months) and late (>12 months) time intervals of operative revision were not statistically significant; all scores were comparable $(p \geq .05)$ - see Table 3. Further analysis of follow-ups in the mid-term (two - five years) and the long-term (more then five years) did not yield statistically significant results $(p \geq .05)$ - see Table 4 .

\section{Radiological findings and their functional correlations}

No implant dislocations and no glenoid loosenings were observed. In one patient, who suffered from rheumatoid arthritis, a loosening of the humeral component had to be evaluated.

Radiographic changes of the tubercles were observed in total in $28 \%(n=19 / 68)$ of patients. A significant increase was observed in the PRSA group in comparison with the SRSA group $(40 \%\{n=17 / 42\}$ vs. $8 \%\{n=2 / 26\}$; $p=.006, \rho=-0.329$ ). See Table 5 for full details. When comparing the healed with unhealed tubercles, statistically significant superiorities were documented in anatomically healed cases in all scores. (all: $p=<0.05$ ). The ROM analysis verified a significant negative ER in unhealed cases $(p=.019)$; see Table 6 for details.

A total rate of scapular notching of $23 \%(n=16 / 68)$ was found without any correlation in the PRSA or SRSA

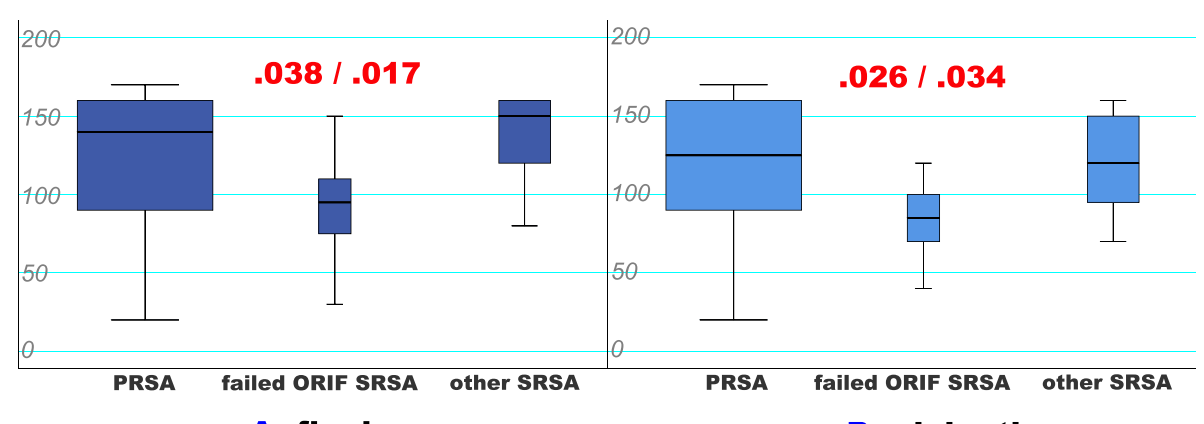

A. flexion

B. abduction

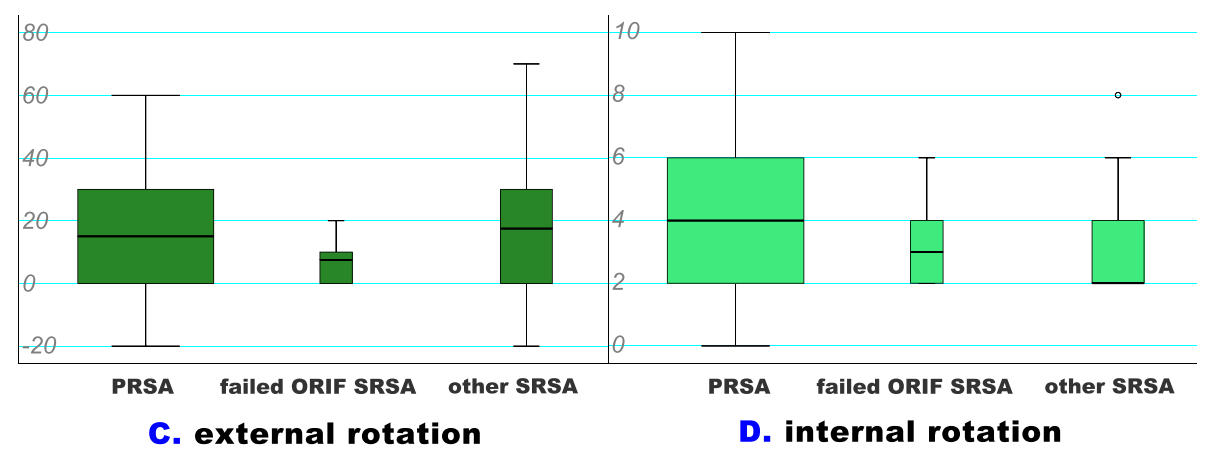

Fig. 6 a-d - ROM analyses for the PRSA and SRSA subgroups: The Kruskal-Wallis test provided strong evidence of differences between the mean ranks of the three groups in $(\mathbf{a})$ flexion $(p=.020)$ and $(\mathbf{b})$ abduction $(p=.020)$. When results were adjusted using the Dunn-Bonferroni method, there was strong evidence of a difference between failed ORIF SRSA and PRSA $(p=.038)$ and failed ORIF SRSA and other SRSA ( $p=.017)$ in terms of flexion. Similarly, there was strong evidence of a difference between failed ORIF SRSA and PRSA $(p=.026)$ and failed ORIF SRSA and other SRSA $(p=.034)$ in terms of abduction. The other results show that there was no statistically significant difference between external (c) and internal rotation (d). Flexion, abduction, and external rotation are displayed in degrees and internal rotation in points according to a sub-item of the Constant score [32]. (failed ORIF SRSA - reverse total shoulder arthroplasty following failed open reduction and internal fixation, PRSA - primary reverse total shoulder arthroplasty, ROM - range of motion, SRSA - secondary reverse total shoulder arthroplasty) 
Table 2 Scores of PRSA versus SRSA

\begin{tabular}{llll}
\hline SCORES & PRSA & SRSA & $P$-value \\
\hline VAS & 1.1 (SD: 2.0) & 0.9 (SD: 1.3) & 0.573 \\
CS & 60 (SD: 16.7) & 56 (SD: 15.1) & 0.204 \\
nCS & 74 (SD: 19.7) & 69 (SD: 18.1) & 0.211 \\
QD & 23 (SD: 20.7) & 25 (SD: 16.7) & 0.291 \\
SSV & $76 \%$ (SD: 18.7) & $75 \%$ (SD: 15.1) & 0.558 \\
\hline
\end{tabular}

Similar results without statistical significance were found for all scores. The SSV is displayed as percentages, and all other scores are shown as points. The average values are presented

(CS absolute Constant Score, $n C S$ normative Constant Score, PRSA primary reverse total shoulder arthroplasty, QD QuickDASH, SD standard deviation, SRSA secondary reverse total shoulder arthroplasty, SSV Subjective Shoulder Value, VAS Visual Analog Scale)

groups $(p=.687, \rho=0.70$; see Table 7$)$. Only one case was classified with a higher grade of notching (grade 3 / PRSA group). No influence of postoperative CS was observed in correlation to notching cases. A mean CS of 55 was evaluated in the notching group. Patients without notching had a CS of 60 on average $(p=$ .352). Similar respective values were shown for in mean nCS and notching versus non-notching: 69 versus $73(p=.268)$.

\section{Complications}

The overall rate of adverse events was $13 \%(n=9 / 68)$. Six percent $(n=4 / 68)$ were classified as major, and seven percent $(n=5 / 68)$ as minor complications. There were fewer complications in the SRSA group when compared with the PRSA group, but this result was not statistically significant $(p=.196)$. The seven complications (in six patients) in the PRSA group included two patients requiring revision surgery. This represents a rate of adverse events of $17 \%$ in the PRSA group $(n=7 / 42)$. The two complications in the SRSA group were a single major and minor complication, which led into one revision surgery. Hence, a rate of $8 \%(n=2 / 26)$ was observed in the SRSA group. The details of all adverse events are listed in Table 8.

\section{Discussion}

The aim of the study was to analyse the effectiveness of the RSA for fracture care in mid-term outcomes via a standardized setting. While RSA significantly improved the treatment of patients with rotator cuff disorders, we focused on the value and better understanding of RSA in fracture management.

The PRSA and SRSA groups did not show statistically significant differences in the range of motion and
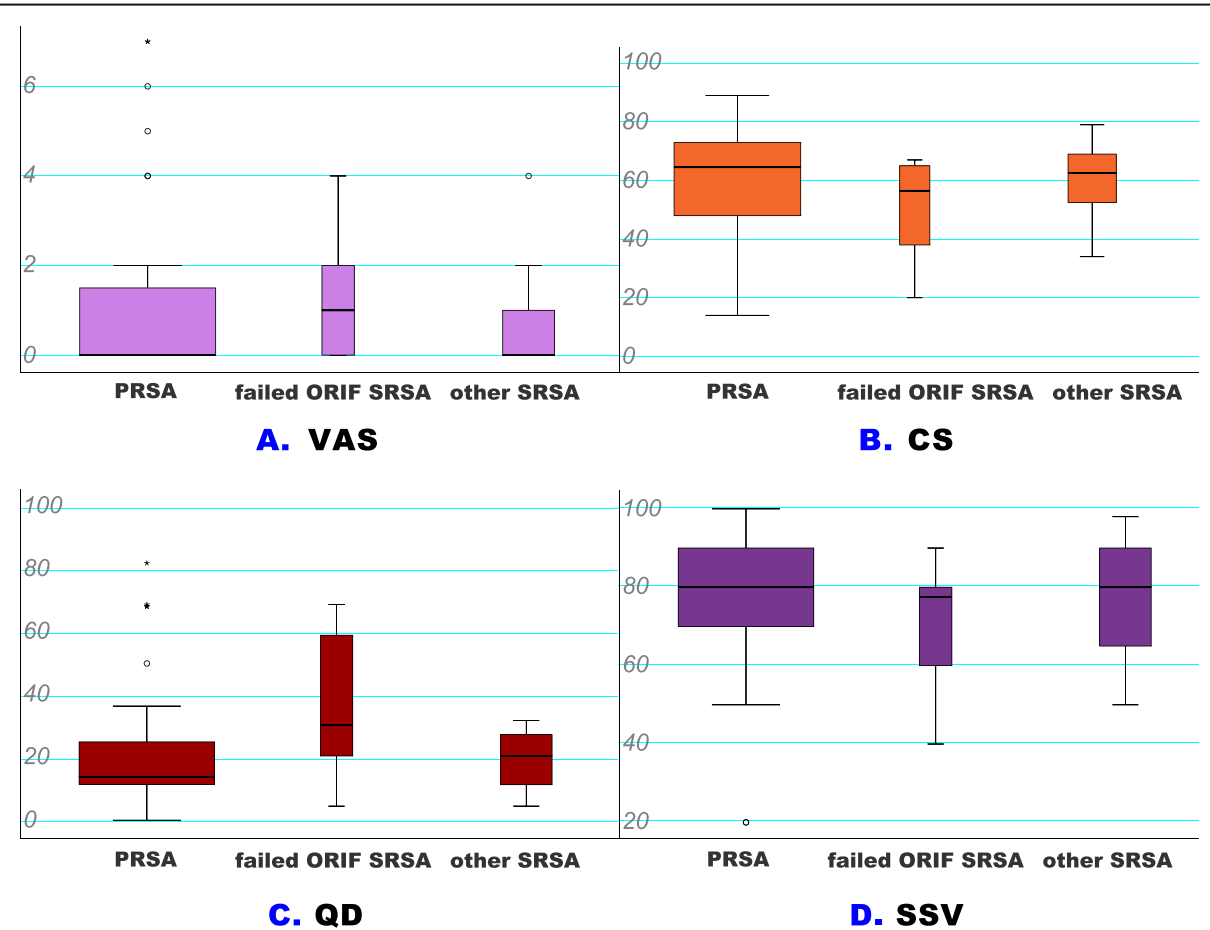

Fig. 7 a-d - Score analyses for the PRSA and SRSA subgroups: The subgroup differentiation revealed no statistical significance and comparable results in all scores (Kruskal-Wallis test and Dunn-Bonferroni method: $p \geq .05$ ). The SSV is displayed as percentages; all other scores are in points. (CS - Constant Score (b), PRSA - primary reverse total shoulder arthroplasty, QD - QuickDASH (c), PRSA - primary reverse total shoulder arthroplasty, SRSA - secondary reverse total shoulder arthroplasty, SSV - Subjective Shoulder Value (d), VAS - Visual Analog Scale(A)) 


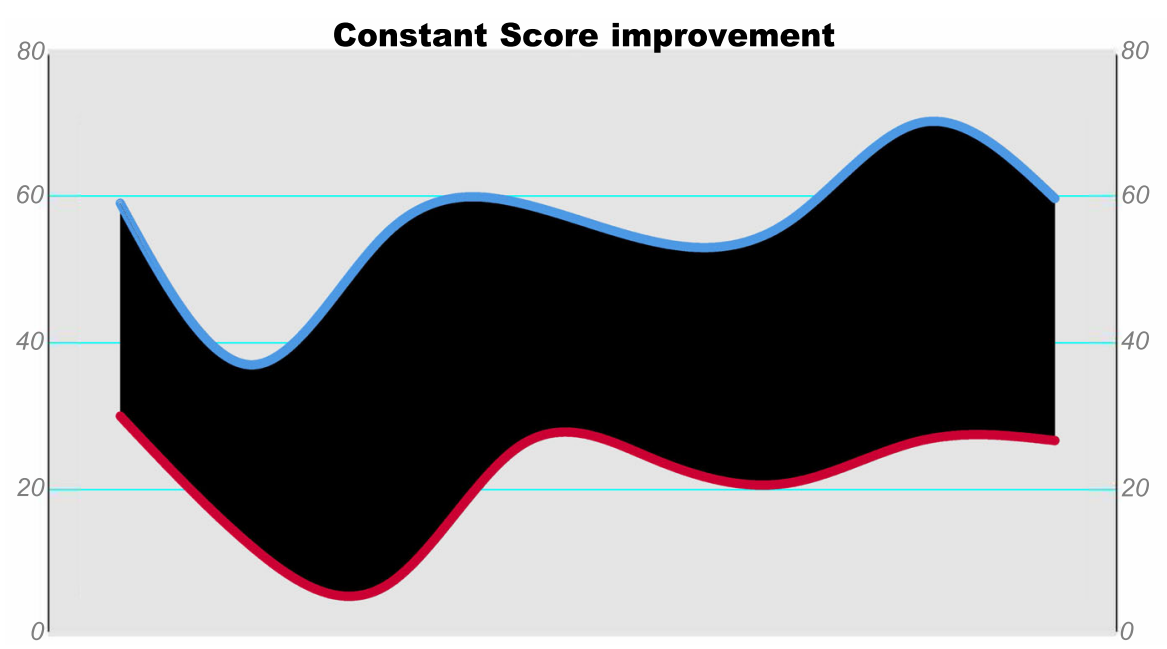

Fig. 8 CS improvement for the SRSA group: The preoperative values to the final follow-up values are displayed in an area graph plot. The red line represents the preoperative values, and the blue line represents the postoperative values of the final control examination. A significant improvement is shown, $p=.001$. (CS - absolute Constant Score, $\mathrm{P}=\mathrm{P}$-value, SRSA - secondary reverse total shoulder arthroplasty)

functional scores, which confirms our hypothesis. Only the subgroup following failed ORIF showed significantly lower values for flexion and abduction when compared with the PRSA group.

Significant improvements between pre- and postoperative CS could be observed in the SRSA group. Furthermore, the time from index to revision surgery had no impact on the functional outcome. Similary, in assessing the longer versus shorter follow-up of the entire collective, no statistically significant decrease in functional findings was observed. These facts indicate that RSA implantation represents a successful treatment strategy in fracture (salvage) care following our standardized operative and postoperative protocol. However, radiographic changes in the tubercles and the overall complication rate were higher in the PRSA group when compared with the SRSA group.

Grubhofer et al. [37] evaluated 51 patients who had undergone RSA for complex proximal humeral fractures

Table 3 Clinical outcomes of early versus late times from index to SRSA surgery

\begin{tabular}{llll}
\hline SCORES & $<\mathbf{1 2}$ MONTHS & $>$ 12 MONTHS & $\boldsymbol{P}$-value \\
\hline VAS & $1.0($ SD: 1.2$)$ & 0.9 (SD: 1.4$)$ & 0.885 \\
CS & 55 (SD: 14.1) & 57 (SD: 16.1$)$ & 0.831 \\
nCS & 68 (SD: 16.8$)$ & 69 (SD: 19.4$)$ & 0.883 \\
QD & 27 (SD: 21.7$)$ & 23 (SD: 13.1$)$ & 0.533 \\
SSV & $75 \%$ (SD: 16.0$)$ & $76 \%(S D: 14.5)$ & 0.882 \\
\hline
\end{tabular}

No statistical relevance was observed; good results were observed in both groups. The SSV is displayed as percentages, and all other scores are shown as points. The average values are presented

(CS Constant Score, $n C S$ normative Constant Score, QD QuickDASH,

$R S A$ reverse total shoulder arthroplasty, SD standard deviation, SRSA secondary reverse total shoulder arthroplasty, SSV Subjective Shoulder Value, VAS Visual Analog Scale) with an average follow-up of 35 months. At the final follow-up check, the absolute CS was at a mean of 62 points, which is well comparable to the PRSA group of our sample (at mean: 60 points).

The French Society of Orthopaedic and Traumatology Surgery [38] performed a pro- and retrospective multicentre study involving nine institutions to investigate RSA outcomes in patients with four-part proximal humeral fractures. The retrospective part of the study included 41 patients with a mean follow-up of 39 months, and the prospective part involved 32 patients with an average follow-up of eleven months. The mean absolute CS was 57 (retrospective) and 50 (prospective) points, respectively. Both values are comparable to our PRSA group (average CS: 60).

Grubhofer and colleagues [39] evaluated 44 shoulders that had undergone revision RSA following unsatisfactory outcomes after proximal humeral ORIF at a mean follow-up of 46 months. The authors reported a

Table 4 Clinical outcomes of longer-term versus mid-term follow-ups

\begin{tabular}{llll}
\hline SCORES & $\mathbf{2 0 0 8 - 2 0 1 3}$ & $\mathbf{2 0 1 4 - 2 0 1 7}$ & $\boldsymbol{P}$-value \\
\hline VAS & $1.3($ SD: 2.1) & 0.8 (SD: 1.5) & 0.319 \\
CS & $55($ SD: 18.6) & $62($ SD: 14.3) & 0.108 \\
nCS & $67(S D: 22.4)$ & $75(S D: 17.2)$ & 0.103 \\
QD & $27($ SD: 22.7) & $21(S D: 16.3)$ & 0.296 \\
SSV & $75 \%(S D: 19.2)$ & $77 \%(S D: 16.0)$ & 0.612
\end{tabular}

No statistical relevance was observed; good functional results were found in both groups. The SSV is displayed as percentages; all other scores are in points. The average values are presented

(CS Constant Score, $n C S$ normative Constant Score, QD QuickDASH,

$R S A$ reverse total shoulder arthroplasty, SD standard deviation, SRSA secondary reverse total shoulder arthroplasty, SSV Subjective Shoulder Value, VAS Visual Analog Scale) 
Table 5 Radiographic tubercle changes in the PRSA and SRSA groups

\begin{tabular}{llll}
\hline LOCALISATION & PRSA & SRSA & $P$-value \\
\hline Major tubercle & $31 \%(n=13 / 42)$ & $11 \%(n=2 / 26)$ & \\
Minor tubercle & & & \\
Both tubercles & $9 \%(n=4 / 42)$ & & \\
TOTAL & $40 \%(n=17 / 42)$ & $8 \%(n=2 / 26)$ & 0.006
\end{tabular}

Statistically significant positive tubercle changes were observed in the PRSA group; radiological changes included dislocations and (partial) resorptions in this group. In the SRSA group, only postoperative partial resorptions were detected. Significant values are shown in bold

(PRSA primary reverse total shoulder arthroplasty, SD standard deviation, SRSA secondary reverse total shoulder arthroplasty)

statistically significant improvement in CS (pre-RSA: 26 [4-54] points; post-RSA: 55 [19-80] points). Their outcome is similar to our failed ORIF SRSA subgroup (mean CS: 52 points). Further, the significant increase of $\mathrm{CS}$ in our results, from a preoperative mean of 23 to a postoperative mean of 56, represents an equivalent effect.

Dezfuli et al. [40] evaluated a sample of 49 patients receiving RSA for either acute proximal humeral fracture, mal- or nonunion, failed ORIF, or trauma hemiarthroplasty. As in our sample, the authors found no statistically significant differences between the subgroups.

Cicak et al. [41] evaluated 37 patients treated with RSA for either acute proximal humeral fractures or sequelae of these. For 21 of these patients, RSA was the primary surgical treatment (14 of these had chronic fracture situations and seven had acute proximal humeral fractures). A further 16 patients had undergone previous surgical therapy, including ORIF or percutaneous

Table 6 - Clinical outcomes of healed versus unhealed tubercles

\begin{tabular}{|c|c|c|c|}
\hline SCORES and ROM & HEALED & UNHEALED & $P$-value \\
\hline$\overline{V A S}$ & 0.7 (SD: 1.3) & 1.6 (SD: 2.5) & 0.162 \\
\hline CS & 62 (SD: 14.1) & 52 (SD: 19.5) & 0.029 \\
\hline$n C S$ & 75 (SD: 17.3) & 64 (SD: 22.7) & 0.021 \\
\hline QD & 19 (SD: 13.4) & 34 (SD: 25.4) & 0.002 \\
\hline SSV & $79 \%$ (SD: 14.4) & $68 \%$ (SD: 21.0 ) & 0.017 \\
\hline Flexion & $129^{\circ}$ (SD: 36.4$)$ & $114^{\circ}$ (SD: 39.6) & 0.125 \\
\hline Abduction & $118^{\circ}$ (SD: 37.7) & $107^{\circ}$ (SD: 35.3$)$ & 0.245 \\
\hline ER & $20^{\circ}(\mathrm{SD}: 19.1)$ & $8^{\circ}(S D: 15.8)$ & 0.019 \\
\hline $\mathbb{R}$ & 4 (SD: 2.2) & 3 (SD: 2.6) & 0.355 \\
\hline
\end{tabular}

Significant positive values were observed for healed tubercles. Flexion, abduction, and ER are displayed in degrees, and IR is reported in points according to a sub-item of the Constant Score [32]. The SSV is displayed in percentages, all other scores are reported in points. Significant results are marked in bold. The average values are presented

(CS Constant Score, ER external rotation, IR internal rotation, $n C S$ normative Constant Score, $Q D$ QuickDASH, ROM range of motion, SD standard deviation, SSV Subjective Shoulder Value, VAS Visual Analog Scale)
Table 7 Grade of scapular notching in the PRSA and SRSA groups

\begin{tabular}{llll}
\hline NOTCHING & PRSA & SRSA & $P$-value \\
\hline Grade 1 & $14 \%(n=6 / 42)$ & $12 \%(n=3 / 26)$ & 0.598 \\
Grade 2 & $5 \%(n=2 / 42)$ & $15 \%(n=4 / 26)$ & 0.684 \\
Grade 3 & $2 \%(n=1 / 42)$ & &
\end{tabular}

Grade 4

In total

$21 \%(n=9 / 42)$

$27 \%(n=7 / 26)$

0.687

Similar notching rates were observed in both groups; this finding was not statistically significant

(PRSA primary reverse total shoulder arthroplasty, SD standard deviation, SRSA secondary reverse total shoulder arthroplasty)

fracture fixation. The group of patients that received RSA for acute fractures had a mean ER of $28^{\circ}$ and an average IR up to the L4 level. In comparison, our PRSA sample had an average ER of $17^{\circ}$ and a mean IR of four points (CS: at L5/S1 level) [32]. The authors reported a mean ER of $19^{\circ}$ and an average IR to L4 for the subgroup that had undergone previous surgery. Our SRSA subgroup showed an ER of $14^{\circ}$ on average and a mean IR of three points (CS: the level between the buttock and iliosacral joint) [32].

Grubhofer et al. [39] evaluated 44 shoulders that had received RSA due to unsatisfactory ORIF and reported an improvement of the preoperative SSV from 29\% (range: $0-90 \%$ ) to $67 \%$ (range: 5-95\%) at the final control examination. We found almost equal values for our PRSA and SRSA groups (76\% and $75 \%$, respectively). The same authors [37] re-evaluated 51 patients (52 shoulders) treated with RSA for acute proximal humeral fractures. The authors reported a mean SSV of $83 \%$ (range: $0-100 \%)$. The French Society of Orthopaedic and Traumatology Surgery [38] reported a mean SSV of $75 \%$ for their retrospective study group and 69\% for their prospective collective. These data are well comparable to our PRSA group, which showed an average SSV of $76 \%$.

Complication rates for RSA are reported to range from 19-68\% [22, 42-44], and include a high percentage of scapular notching and impairment of external rotation as the main problems [18]. Furthermore, RSA may involve periprosthetic fractures, fractures of the glenoid, acromion or humeral shaft, neurological lesions, infection, dislocation, mechanical failures or loosening of the glenosphere [2, 22]. Lehtimäki et al. [2] identified all RSAs utilized for proximal humeral fractures from the Nordic Arthroplasty Register Association registry data for the interval between 2004 and 2016, whereby 1523 implantations were included in the study. Only $2 \%$ of these $(33 / 1523)$ required revision surgery with instability reported as the most common reason (11/1523). The nine adverse effects (13\%) and 6\% major complication rate of the present study have to be re-evaluated. 
Table 8 Adverse events

\begin{tabular}{lllll}
\hline & EVENT & MONTHS & INTERVENTION & CLASSIFICATION \\
\hline 1 & $\begin{array}{l}\text { spontaneous muscle hematoma } \\
\text { (under direct oral anticoagulant) } \\
\text { spontaneous muscle hematoma } \\
\text { (under direct oral anticoagulant) }\end{array}$ & 60 & conservative & minor \\
3 & instability with dislocation & 35 & conservative & minor \\
4 & major tubercle impingement & 10 & inlay change & major \\
5 & traumatic periprosthetic fracture (accident) & 7 & conservative & minor \\
6 & scapular spine fracture & 131 & ORIF & major \\
7 & shaft loosening & 116 & conservative & minor \\
8 & traumatic periprosthetic fracture (fall) & 70 & conservative (non vult) & major \\
9 & plexus neuropraxia & 25 & ORIF & major \\
\hline
\end{tabular}

Major and minor complications of total follow-ups in all patients are displayed. Their time- and event-related specifications are shown. The time from RSA implantation to each adverse event is presented in months. The events numbered from 1 to 7 involved PRSA patients, and events numbered from 8 to 9 involved SRSA patients. Multiple events are possible in one patient

(ORIF open reduction and internal fixation, PRSA primary reverse total shoulder arthroplasty, RSA reverse total shoulder arthroplasty, SRSA secondary reverse total shoulder arthroplasty)

Implant-related major complications were present in only one case and were soley due to instability. A shaft loosening had to be attributed to an underlying disease. All other adverse events were not implant-related or fateful events. These facts lead to a major complication rate of $3 \%$ in our total collective, comparable to the findings of Lehtimäki et al. [2] in a sample with a high number of included patients. Moreover, the rate of adverse events was similar in primary and secondary care.

Common agreements exist regarding decreasing notching rates in modern prosthesis designs [45]. The clinical impact of scapular notching appears to be controversial in the literature. Some authors report a significant decrease in outcomes by notching; others declare no significant influence [46-48]. A recent systematic review [49], including 2,222 shoulder arthroplasties, found that $155^{\circ}$ implants had a total notching rate of $16.8 \%$. The authors reported that the notching rate was significantly higher in the $155^{\circ}$ design than in the $135^{\circ}$ design. These values are comparable to our results for a $23 \%$ notching rate.

Jain et al. [50] identified a tubercle healing rate of $70.5 \%$, which is equivalent to our results of $72 \%$ in a meta-analysis of 382 shoulder arthroplasties and a similar follow-up period. Acceptable functional results in unhealed tubercle patients ( $28 \%$ of the total collective) substantiate that a fracture RSA implantation allows predictable postoperative results. Nevertheless, statistically significant superiorities for healed tubercles were shown in all functional scores and patient satisfaction characteristics, as well as in ER - an essential aspect for the daily processes. Based on these data for mid-term clinical outcomes, we recommend that surgeons focus on tubercle refixation.

\section{Strengths and limitations}

This study investigated the combined effect of radiological events and tubercle bone stock associated with clinical outcomes in primary and secondary fracture care and allowed preoperative assessments in order to estimate postoperative processes. Due to changes in patient requirements and the increasing demand for salvage procedures, this study reinforces our understanding with mid-term results in an assimilable patient number. The clinical significance is fortified by the continuously increasing implantation rates of RSA in fracture care in modern material designs. Future research is needed to establish potential superiorities and the best surgical options for younger patients.

However, there are several limitations to this study. First, the study design was retrospective in nature, and our preoperative documentation did not collect all the tests and scores used in our follow-up study (with the exception of the SRSA group). Therefore, amelioration only applies to the CS and $\mathrm{nCS}$ in the SRSA group.

Second, our follow-up period was limited, and the complication rate might increase with time. Next, group sizes in number of patients, implant design and follow-up varied. This aspect is comparable to the literature data in this patient sample. Furthermore, the study was conducted using only reverse total shoulder systems with cemented humeral components, and direct comparisons to their contemporaries the cementlesses RSA systems, cannot be drawn. Lastly, multiple surgeons (four experienced trauma and orthopaedic surgeons) were involved, and no comparisons regarding their personal experiences and outcomes were performed. 


\section{Conclusions}

The similar mid-scale/-term findings of both strategies confirm the value of RSA in complex shoulder fracture management as primary or secondary / salvage care to maintain autonomy. Predictable, promising mid-term functional results, high patient satisfaction, and excellent pain relief could be demonstrated.

In SRSA, significant postoperative improvements could be observed. The time from index surgery to operative revision was not a no statistically significant factor for clinical outcomes.

A satisfactory total tubercle healing rate was proven, since a significant increase of radiographic changes was shown in the PRSA group. Statistically significant improvements in objective and subjective findings for healed tubercles were shown. Further, no statistically significant correlations were observed for notching in the PRSA or SRSA groups in the modern material design.

In our setting, RSA has become a successful and effective option for complex proximal humeral cases with inadequate bone stock. This non-joint-preserving management burdened by a low complication rate and a reasonable implant survival rate, whereby a specific patient selection should occur due to the few available options in cases of RSA failure.

\section{Abbreviations \\ AUVA: Austrian Social Insurance for Occupational Risks; CS: absolute Constant Score; ER: external rotation; IR: internal rotation; nCS: normative Constant Score; ORIF: open reduction and internal fixation; QD: QuickDASH; $p$ : $p$-value; PRSA: primary reverse total shoulder arthroplasty; $\rho$ : Spearman's Rho correlation coefficient; ROM: range of motion; RSA: reverse total shoulder arthroplasty; SANE: Single Assessment Numeric Evaluation; SD: standard deviation; SRSA: secondary reverse total shoulder arthroplasty; SSV: Subjective Shoulder Value; VAS: Visual Analog Scale}

\section{Acknowledgements}

The authors acknowledge and thank the participants and the team of the AUVA - Trauma Hospital (UKH) Styria | Graz for their help and support during the study.

\section{Authors' contributions}

AS was responsible for the initiation of the study and analysis of the data as well as the first draft of the manuscript and contributed significantly, with the input from MP, to the final draft of the manuscript. MP had the original idea for the study, initiated the study and participated in critical revisions of the article for important intellectual content and supervision. AS and MP developed the study design, and all authors conceived the study protocol. AS, GH, MS, MN, and MP contributed to the acquisition and assembly of data, data management, and analysis. AS, GH and MN performed the radiological assessment. GL, GM, MZ, and FS contributed to the interpretation of the results and the manuscript development. All authors were involved in drafting of the manuscript and approved the final version to be submitted for publication.

\section{Authors' informations}

Not applicable.

\section{Funding}

The authors declare that there was no funding source.

\section{Availability of data and materials}

The datasets used and/or analysed during the current study are available from the corresponding author upon reasonable request.

\section{Ethics approval and consent to participate}

The study protocol was approved by the local ethics committee (Votum EK 32/2017) of the Austrian Social Insurance for Occupational Risks (AUVA), and was performed in agreement with the protocol. The study was performed in accordance with the principles of the Declaration of Helsinki and the $\mathrm{ICH}$ GCP Guidelines. Written informed consent was obtained from all individual participants included in the study.

\section{Consent for publication}

Not applicable.

\section{Competing interests}

The authors declare that they have no competing interests.

\section{Author details}

${ }^{1}$ AUVA - Trauma Hospital (UKH) Styria | Graz, Teaching Hospital of the Medical University of Graz, Göstinger Straße 24, 8020 Graz, Austria.

${ }^{2}$ Department of Orthopaedics and Trauma, Medical University of Graz, Graz, Austria.

Received: 22 August 2020 Accepted: 21 December 2020

Published online: 08 January 2021

\section{References}

1. García-Fernández C, Lopiz Y, Rizo B, Serrano-Mateo L, Alcobía-Díaz B, Rodríguez-González A, et al. Reverse total shoulder arhroplasty for the treatment of failed fixation in proximal humeral fractures. Injury. 2018;49: S22-6. Available from: https://doi.org/10.1016/j.injury.2018.06.042.

2. Lehtimäki K, Rasmussen JV, Kukkonen J, Salomonsson B, Arverud ED, Hole R, et al. Low risk of revision after reverse shoulder arthroplasty for acute proximal humeral fractures. JSES Int. 2020;4(1):151-5.

3. Ehlinger M, Gicquel P, Clavert P, Bonnomet F, Kempf J-F. Un nouvel implant pour les fractures de l'humérus proximal: la plaque à corbeille. Rev Chirurgie Orthop Rép I'Appareil Moteur. 2004;90(1):16-25.

4. Konrad G, Bayer J, Hepp P, Voigt C, Oestern H, Kääb M, et al. Open reduction and internal fixation of proximal humeral fractures with use of the locking proximal humerus plate: Surgical technique. J Bone Joint Surg Series A. 2010;92(SUPPL. 1 PART 1):85-95.

5. Kristiansen B, Christensen SW. Plate fixation of proximal humeral fractures. Acta Orthop. 1986;57(4):320-3.

6. Plecko M, Kraus A. Internal Fixation of Proximal Humerus Fractures Using the Locking Proximal Humerus Plate (LPHP). Operative Orthop Traumatol. 2005 Feb;17(1):25-50. Available from: http://link.springer.com/https://doi. org/10.1007/s00064-005-1120-8.

7. Laux CJ, Grubhofer F, Werner CML, Simmen HP, Osterhoff G. Current concepts in locking plate fixation of proximal humerus fractures. Vol. 12, J Orthop Surg Res. 2017. p. 137. Available from: https://www.ncbi.nlm.nih. gov/pubmed/28946902.

8. Agel J, Jones CB, Sanzone AG, Camuso M, Henley MB. Treatment of proximal humeral fractures with Polarus nail fixation. J Shoulder Elbow Surg. 2004;13(2):191-5.

9. Dilisio MF, Nowinski RJ, Hatzidakis AM, Fehringer EV. Intramedullary nailing of the proximal humerus: Evolution, technique, and results. J Shoulder Elbow Surg. 2016;25(5):e130-8. Available from: https://doi.org/10.1016/j.jse. 2015.11.016.

10. Boileau P, Trojani C, Walch G, Krishnan SG, Romeo A, Sinnerton R. Shoulder arthroplasty for the treatment of the sequelae of fractures of the proximal humerus. J Shoulder Elbow Surg. 2001;10(4):299-308.

11. Jacobson JA, Duquin TR, Sanchez-Sotelo J, Schleck CD, Sperling JW, Cofield $\mathrm{RH}$. Anatomic shoulder arthroplasty for treatment of proximal humerus malunions. J Shoulder Elbow Surg. 2014;23(8):1232-9. Available from: https://doi.org/10.1016/j.jse.2013.11.015.

12. Mansat P, Bonnevialle N. Treatment of fracture sequelae of the proximal humerus: anatomical vs reverse shoulder prosthesis. Int Orthop. 2015;39(2): 349-54. 
13. Kiliç M, Berth A, Blatter G, Fuhrmann U, Gebhardt K, Rott O, et al. Anatomic and reverse shoulder prostheses in fracture sequelae of the humeral head. Acta Orthop Traumatol Turcica. 2010;44(6):417-25.

14. Giovale M, Mangano T, Rodà E, Repetto I, Cerruti P, Kuqi E, et al. Shoulder hemiarthroplasty for complex humeral fractures: A 5 to 10-year follow-up retrospective study. Musculoskelet Surg. 2014;98(SUPPL. 1):27-33.

15. Baudi P, Campochiaro G, Serafini F, Gazzotti G, Matino G, Rovesta C, et al. Hemiarthroplasty versus reverse shoulder arthroplasty: Comparative study of functional and radiological outcomes in the treatment of acute proximal humerus fracture. Musculoskelet Surg. 2014;98(SUPPL. 1).

16. lacobellis C, Berizzi A, Biz C, Camporese A. Treatment of proximal humeral fractures with reverse shoulder arthroplasty in elderly patients. Musculoskelet Surg. 2015;99(1):39-44.

17. Klein M, Juschka M, Hinkenjann B, Scherger B, Ostermann PAW. Treatment of comminuted fractures of the proximal humerus in elderly patients with the delta III reverse shoulder prosthesis. J Orthop Trauma. 2008;22(10):698-704.

18. Russo R, Della Rotonda G, Cautiero F, Ciccarelli M. Reverse shoulder prosthesis to treat complex proximal humeral fractures in the elderly patients: results after 10-year experience. Musculoskelet Surg. 2015;99:17-23.

19. Boyle MJ, Youn SM, Frampton CMA, Ball CM. Functional outcomes of reverse shoulder arthroplasty compared with hemiarthroplasty for acute proximal humeral fractures. J Shoulder Elbow Surg. 2013;22(1):32-7. Available from: https://doi.org/10.1016/j.jse.2012.03.006.

20. Naranja RJ, lannotti JP. Displaced three- and four-part proximal humerus fractures: evaluation and management. J Am Acad Orthop Surg. 2000;8(6):373-82.

21. Savin DD, Zamfirova I, lannotti J, Goldberg BA, Youderian AR. Survey study suggests that reverse total shoulder arthroplasty is becoming the treatment of choice for four-part fractures of the humeral head in the elderly. Int Orthop. 2016;40(9):1919-25. Available from: https://doi.org/10.1007/s00264-016-3227-y.

22. Familiari F, Rojas J, Doral MN, Huri G, McFarland EG. Reverse total shoulder arthroplasty. EFORT Open Rev. 2018;3(2):58-69.

23. Flatow EL, Harrison AK. A history of reverse total shoulder arthroplasty. Clin Orthop Relat Res. 2011;469(9):2432-9.

24. Baulot E, Sirveaux F, Boileau P. Grammont's, Idea: The Story of Paul Grammont's Functional Surgery Concept and the Development of the Reverse Principle. Clinical Orthopaedics and Related Research ${ }^{\oplus} .2011$ Sep 6; 469(9):2425-31. Available from: http://link.springer.com/https://doi.org/10. 1007/s11999-010-1757-y.

25. Grammont PM, Baulot E. Delta shoulder prosthesis for rotator cuff rupture. Vol. 16, Orthopedics. 1993. p. 65-8. Available from: https://pubmed.ncbi.nlm. nih.gov/8421661/.

26. Naveed MA, Kitson J, Bunker TD. The Delta III reverse shoulder replacement for cuff tear arthropathy: A single-centre study of 50 consecutive procedures. Journal of Bone Joint Surgery - Series B. 2011;93 B(1):57-61.

27. Smith CD, Guyver $P$, Bunker TD. Indications for reverse shoulder replacement. Journal of Bone Joint Surgery - Series B. 2012;94 B(5):577-83.

28. Anakwenze OA, Zoller S, Ahmad CS, Levine WN. Reverse shoulder arthroplasty for acute proximal humerus fractures: A systematic review. Journal of Shoulder and Elbow Surgery [Internet]. 2014;23(4):e73-80. Available from: https://doi.org/10.1016/j.jse.2013.09.012.

29. Patel DN, Young B, Onyekwelu I, Zuckerman JD, Kwon YW. Reverse total shoulder arthroplasty for failed shoulder arthroplasty. Journal of Shoulder and Elbow Surgery [Internet]. 2012;21(11):1478-83. Available from: https:// doi.org/10.1016/j.jse.2011.11.004.

30. Neer CSI. Displaced Proximal Humeral Fractures: PART I. CLASSIFICATION. J Bone Joint Surg Am. 1970;(52):1077-89.

31. Neer CS. Four-segment classification of proximal humeral fractures: Purpose and reliable use. J Shoulder Elbow Surg. 2002;11(4):389-400.

32. Constant $\mathrm{CR}$, Murley AHG. A clinical method of functional assessment of the shoulder. Clinical Orthopaedics and Related Research. 1987;NNo. 214(April):160-4.

33. Habermeyer $P$, Magosch $P$, Lichtenberg S. Classifications and scores of the shoulder. Classifications and Scores of the Shoulder. 2006. 1-297 p.

34. Gilbart MK, Gerber C. Comparison of the subjective shoulder value and the Constant score. J Shoulder Elbow Surg. 2007;16(6):717-21.

35. Beaton DE, Wright JG, Katz JN, Amadio P, Bombardier C, Cole D, et al. Development of the QuickDASH: COmparison of three item-reduction approaches. J Bone Joint Surg Series A. 2005;87(5):1038-46.

36. Sirveaux F, Favard L, Oudet D, Huquet D, Walch G, Molé D. Grammont inverted total shoulder arthroplasty in the treatment of glenohumeral osteoarthritis with massive rupture of the cuff. Results of a multicentre study of 80 shoulders. J Bone Joint Surg Series B. 2004;86(3):388-95.
37. Grubhofer F, Wieser K, Meyer DC, Catanzaro S, Beeler S, Riede U, et al. Reverse total shoulder arthroplasty for acute head-splitting, 3- and 4-part fractures of the proximal humerus in the elderly. Journal of Shoulder and Elbow Surgery. 2016;25(10):1690-8. Available from: https://doi.org/10.1016/j.jse.2016.02.024.

38. Obert L, Saadnia R, Tournier C, Bonnevialle N, Saragaglia D, Sirveaux F. Fourpart fractures treated with a reversed total shoulder prosthesis: Prospective and retrospective multicenter study. Results and complications. Orthopaedics and Traumatology: Surgery and Research. 2016;102(3):279-85.

39. Grubhofer F, Wieser K, Meyer DC, Catanzaro S, Schürholz K, Gerber C. Reverse total shoulder arthroplasty for failed open reduction and internal fixation of fractures of the proximal humerus. Journal of Shoulder and Elbow Surgery. 2017;26(1):92-100. Available from: https://doi.org/10.1016/j.jse.2016.05.020.

40. Dezfuli B, King JJ, Farmer KW, Struk AM, Wright TW. Outcomes of reverse total shoulder arthroplasty as primary versus revision procedure for proximal humerus fractures. Journal of Shoulder and Elbow Surgery. 2016;25(7):11337. Available from: https://doi.org/10.1016/j.jse.2015.12.002.

41. Cicak N, Klobucar H, Medancic N. Reverse shoulder arthroplasty in acute fractures provides better results than in revision procedures for fracture sequelae. International Orthopaedics. 2015;39(2):343-8. Available from: http://link.springer.com/https://doi.org/10.1007/s00264-014-2649-7.

42. Cheung E, Willis M, Walker M, Clark R, Frankle MA. Complications in Reverse Total Shoulder Arthroplasty. JAAOS - Journal of the American Academy of Orthopaedic Surgeons. 2011;19(7). Available from: https://journals.lww.com/ jaaos/Fulltext/2011/07000/Complications_in_Reverse_Total_Shoulder.7.aspx.

43. Zumstein MA, Pinedo M, Old J, Boileau P. Problems, complications, reoperations, and revisions in reverse total shoulder arthroplasty: A systematic review. Journal of Shoulder and Elbow Surgery. 2011;20(1):14657. Available from: https://doi.org/10.1016/j.jse.2010.08.001.

44. Barco R, Savvidou OD, Sperling JW, Sanchez-Sotelo J, Cofield RH. Complications in reverse shoulder arthroplasty. EFORT Open Rev. 2016;1(3): 72-80 Available from: www.efort.org/openreviews.

45. Taliaferro JP, Crosby LA. Scapular notching in reverse total shoulder arthroplasty: Now what? Seminars in Arthroplasty. 2014;25(1):44-7. Available from: https://doi.org/10.1053/j.sart.2014.02.008

46. Mollon B, Mahure SA, Roche CP, Zuckerman JD. Impact of scapular notching on clinical outcomes after reverse total shoulder arthroplasty: an analysis of 476 shoulders. J Shoulder Elbow Surg. 2017;26(7):1253-61. Available from: https://doi.org/10.1016/j.jse.2016.11.043.

47. Alentorn-Geli E, Samitier G, Torrens C, Wright TW. Reverse shoulder arthroplasty. Part 2: Systematic review of reoperations, revisions, problems, and complications. Int J Shoulder Surg. 2015;9(2):60-7.

48. Samitier G, Alentorn-Geli E, Torrens C, Wright TW. Reverse shoulder arthroplasty. Part 1: Systematic review of clinical and functional outcomes. Int J Shoulder Surg. 2015:9(1):24-31.

49. Erickson BJ, Frank RM, Harris JD, Mall N, Romeo AA. The influence of humeral head inclination in reverse total shoulder arthroplasty: A systematic review. J Shoulder Elbow Surg. 2015;24(6):988-93. Available from: https:// doi.org/10.1016/j.jse.2015.01.001.

50. Jain NP, Mannan SS, Dharmarajan R, Rangan A. Tuberosity healing after reverse shoulder arthroplasty for complex proximal humeral fractures in elderly patients_-does it improve outcomes? A systematic review and meta-analysis. J Shoulder Elbow Surg. 2019;28(3):e78-91. Available from: https://doi.org/10.1016/j.jse.2018.09.006.

\section{Publisher's Note}

Springer Nature remains neutral with regard to jurisdictional claims in published maps and institutional affiliations.

\section{Ready to submit your research? Choose BMC and benefit from:}

- fast, convenient online submission

- thorough peer review by experienced researchers in your field

- rapid publication on acceptance

- support for research data, including large and complex data types

- gold Open Access which fosters wider collaboration and increased citations

- maximum visibility for your research: over $100 \mathrm{M}$ website views per year

At $\mathrm{BMC}$, research is always in progress.

Learn more biomedcentral.com/submissions 